\title{
Characterizations of biodegradable epoxy-coated cellulose nanofibrils (CNF) thin film for flexible microwave applications
}

\author{
Hongyi Mi • Chien-Hao Liu • Tzu-Husan Chang • Jung-Hun Seo • \\ Huilong Zhang $\cdot$ Sang June Cho $\cdot$ Nader Behdad $\cdot$ Zhenqiang Ma $•$ \\ Chunhua Yao $\cdot$ Zhiyong Cai $\cdot$ Shaoqin Gong
}

Received: 19 September 2015/Accepted: 18 March 2016/Published online: 28 March 2016

(C) The Author(s) 2016. This article is published with open access at Springerlink.com

\begin{abstract}
Wood pulp cellulose nanofibrils (CNF) thin film is a novel recyclable and biodegradable material. We investigated the microwave dielectric properties of the epoxy coated-CNF thin film for potential broad applications in flexible high speed electronics. The characterizations of dielectric properties were carried out in a frequency range of 1-10 GHz. The dielectric constant and loss tangent were extracted by using measurements of microstrip transmission lines that were built on the epoxy coatedCNF film and combined with Agilent Advanced Design System simulations. The RF property for the epoxy coated-CNF was compared to that of commercial polyethylene terephthalate film (PET). With the comparable microwave properties of non-biodegradable PET film, our study suggests that the epoxy
\end{abstract}

H. Mi · C.-H. Liu - T.-H. Chang - J.-H. Seo ·

H. Zhang $\cdot$ S. J. Cho $\cdot$ N. Behdad $\cdot$ Z. Ma $(\bowtie)$

Department of Electrical and Computer Engineering,

University of Wisconsin-Madison, 1415 Engineering

Drive, Madison, WI 53706, USA

e-mail: mazq@engr.wisc.edu

C. Yao $\cdot$ Z. Cai $(\bowtie)$

USDA Forest Products Laboratory, One Gifford Pinchot Drive, Madison, WI 53726, USA

e-mail: zcai@fs.fed.us

S. Gong

Department of Biomedical Engineering, Wisconsin Institute for Discovery, University of Wisconsin-Madison, 330 North Orchard Street, Madison, WI 53715, USA
coated-CNF film is a suitable biodegradable and environment-friendly substrate for flexible microwave and other applications.

Keywords Dielectric characterization - Epoxy coated-cellulose nanofibrils (CNF) - Biodegradable . Transmission line measurement $\cdot$ Flexible substrate . RF loss

\section{Introduction}

In recent years, flexible electronics have been rapidly developed for many applications. In particular, RF and microwave devices and circuitry have been designed and fabricated on flexible substrates, such as polyethylene terephthalate (PET), to work in the giga-hertz frequency range (Zhang et al. 2012; Seo et al. 2014). These demonstrations indicate flexible electronics can not only fulfill traditional low frequency applications, but also RF and microwave applications with highperformance flexible microwave active (Sun et al. 2010b; Zhou et al. 2013) and passive devices (Sun et al. 2010a; Cho et al. 2015). It is expected the future production of flexible electronics will greatly exceed that of rigid chip-based electronics. While over 3 million tons of electronic (e-) waste per year has piled up over the last decade due to rapid upgrades of consumer electronics (Lorenzen 2014), more e-waste is expected to be produced as we enter the era of 
flexible electronics. The majority of today's polymer based substrates used for flexible electronics and packaging (Zou et al. 2002; Thompson et al. 2004) are made of chemical materials. These petroleum based materials are rather difficult to decompose after disposal into the e-waste stream. To reduce this accumulation of e-waste, flexible electronics with substrates made of decomposable materials are preferred. Wood pulp composites [such as cellulose nanofibril (CNF)] are one group of recyclable materials that exhibit impressive biodegradable properties (Chinga-Carrasco 2011; Abdul Khalil et al. 2012). The outstanding characteristics of wood pulp composite films, such as high mechanical strength and high thermal conductivity (Bledzki et al. 1998; Zhang et al. 2013), have made them promising candidates for substrates of flexible electronics (Zheng et al. 2013). Although there are some demonstrations using these composites for micro-electro-mechanical system (Couderc et al. 2009) and microwave applications (Inui et al. 2015; Jung et al. 2015; Seo et al. 2015), detailed characterizations of microwave properties for these materials have not been previously reported. In this paper, we report the detailed study of frequencydependent microwave properties of the epoxy coatedcellulose nanofibril (CNF) film.

\section{Experiment}

Epoxy coat-CNF film fabrication

The epoxy coated-CNF film fabrication began with tetramethylpiperidine-1-oxy (TEMPO) oxidized wood pulp fiber (Isogai et al. 2011), which was prepared using the method reported (Qing et al. 2012). The TEMPO oxidized cellulose fibers were then refined in a Microfluidizer processor (Microfluidics, Newton, MA). The obtained nanofiber slurry (Fig. 1a), diluted to a $0.2 \%$ solid concentration by DI water, was filtrated using a $142 \mathrm{~mm}$ Milipore Ultrafiltration System (Millipore, Millipore Corporation, USA; Fig. 1b) under 0.55 MPa air pressure. An Omnipore $^{\mathrm{TM}}$ polytetrafluoroethylene filter membrane with micropore size of $0.1 \mu \mathrm{m}$ (JVWP14225, JV, Millipore Corporation, USA) was used in the apparatus and supported by filter paper. In the filtration chamber, water within the slurry passed through the filter membrane, leaving the CNF filter cake. After separated from the filter membrane, the CNF cake was sandwiched between two multiple layers of waxy coated paper, filter paper, and caul plate at room temperature for drying. Throughout the drying process, those papers were replaced several times to avoid winkles on the film. When the film approximately reached a solid content of $80-90 \%$, the sandwich assembly was put into oven for several hours drying at $60{ }^{\circ} \mathrm{C}$ for $8 \mathrm{~h}$ under a load of $250 \mathrm{~N}$. The dried CNF film was coated with a bisphenol-A-based epoxy resin kit (Dow Chemical Company). The low viscosity epoxy resin (D.E.R. 331), flexible epoxy resin (D.E.R. 732) and amine-based curing agent (D.E.H. 26) were mixed by a weight ratio of 56:24:20, respectively. This epoxy mixture was brushed onto one side of the CNF film, then pressed under $100 \mathrm{psi}$ at $130{ }^{\circ} \mathrm{C}$ for $10 \mathrm{~min}$, followed by processing on the other side of the CNF film in the same way. Then, the film was subsequently covered with a plastic sheet, and a cylinder was rolled over the sheet. The rolling was repeated until the cellulose film was completely and uniformly covered with the epoxy. After the above apparatus was air dried, the plastic sheet was peeled off, and an epoxy coated-CNF film was obtained. The epoxy coatedCNF film was transparent, flexible, very durable, and biodegradable with a thickness of $180-190 \mu \mathrm{m}$ as shown in Fig. 1c. Figure 1d shows an optical image of an epoxy coated-CNF film after partial fungal biodegradation. Besides biodegradability, the film also exhibited great thermal stability, electrical stability, and mechanical properties (Jung et al. 2015), which qualify the substrate for flexible electronics applications. The epoxy we used in this experiment has similar dielectric and RF loss properties to that of CNF films in the microwave frequency range of interest under standard room humidity conditions (Minges 1989; Torgovnikov 2012). Therefore, the microwave dielectric properties of CNF films with epoxy coating will remain unchanged. Additionally, the hydrophobic characteristic of the epoxy helps prevent the CNF films from absorbing moisture from the external environment and thus maintain the dielectric properties (Couderc et al. 2009). It is noted that the epoxy coated-CNF films still can be degraded by the common fungi. The experimental characterizations of the dielectric properties of the epoxy coated$\mathrm{CNF}$ film were carried out at $22{ }^{\circ} \mathrm{C}$ under $35 \%$ humidity in the frequency range of $1-10 \mathrm{GHz}$, which covers the wireless communication bands: the $L, S$, 

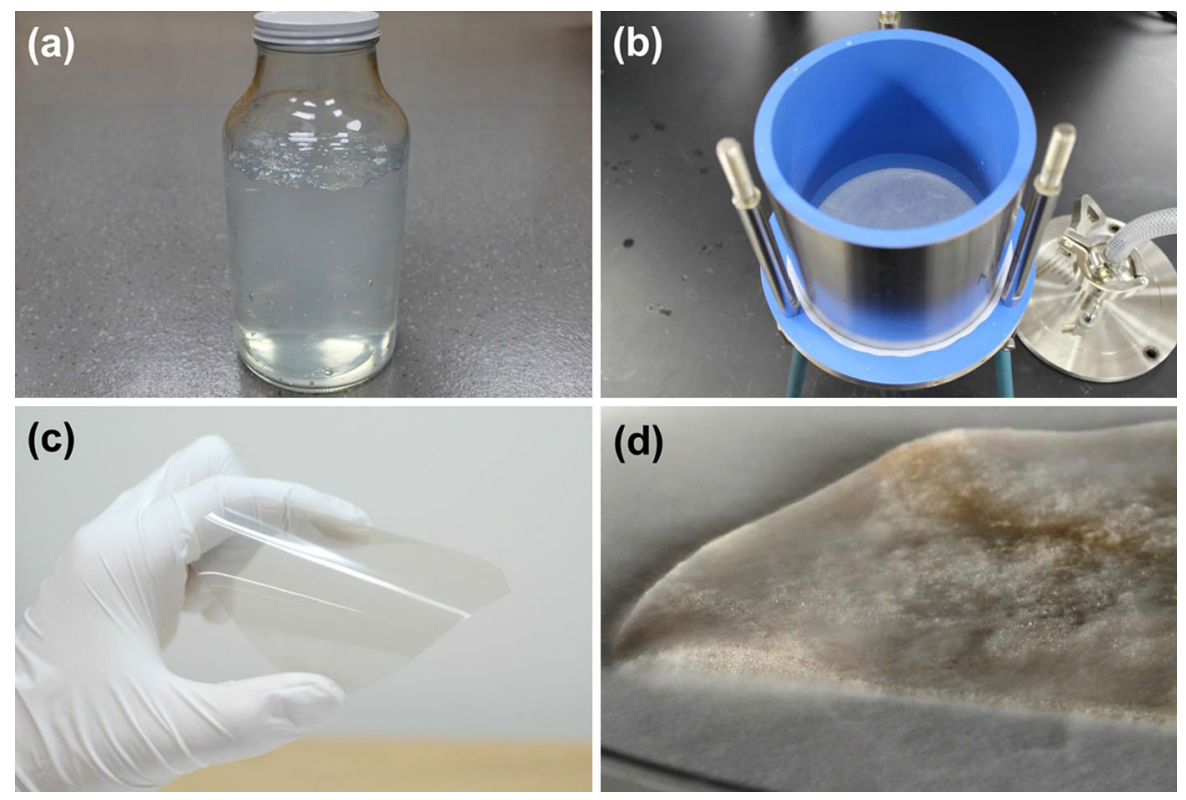

Fig. 1 a An optical image of the obtained nanofiber slurry. b An optical image of the Milipore ultrafiltration system. c Obtained flexible epoxy coated-CNF film under bending. $\mathbf{d}$ An optical image of the epoxy coated-CNF film after fungal biodegradation

$C$ and the lower part of the $X$-bands. For the purpose of comparison, we also performed identical experiments on commercial PET films.

Transmission line method characterizations

The transmission line method (TLM) is suitable for characterizing microwave dielectric properties of thin films in a wide frequency range (Moon-Que Lee 1996; Baker-Jarvis et al. 2010; Aguilar et al. 2012). In this method, thin film is adopted as the substrate for microstrip transmission lines. The dielectric properties of the thin film can be extracted from measured scattering parameters (S-parameters). This method was employed to characterize the microwave dielectric properties of the epoxy coated-CNF film in the frequency range of 1-10 GHz. The transmission (signal) line, made of $100 \mu \mathrm{m}$-thick copper foil tape that was built on the CNF substrate, was about $42 \mathrm{~mm}$ long and $6 \mathrm{~mm}$ wide. It was made to cover the entire length of the epoxy coated-CNF substrate and was chosen to be long enough for several guided wavelengths, in order to reduce the influence of the SMA connectors and parasitic effects between the connector and transmission line, on overall measurement accuracy. The ground plane of the microstrip transmission line was made by covering the backside of the epoxy coated-CNF substrate with a conducting copper foil tape. A flat copper sheet was then brought into intimate contact with the copper foil tape to facilitate mounting of the SMA connectors. Two SMA connectors were finally soldered to the structure. The schematic of a microstrip transmission line and the fabricated transmission line are shown in Fig. 2. The two port S-parameters of the assembled microstrip transmission line were measured in the frequency range of $1-10 \mathrm{GHz}$ using an Agilent E8364A performance network analyzer.

An equivalent circuit model for this microstrip transmission line was built using Agilent/Keysight Advanced Design System (ADS). This circuit also included the presence of an edge mounted SMA connector on each side and illustrated parasitic effects between the connectors and microstrip transmission line. The circuit structure is shown in Fig. 3. ADS simulations were performed to identify the equivalent circuit parameters that fit experimentally measured values (Ramazani et al. 2010; Aguilar et al. 2012). The dielectric constant and loss tangent of the substrate material were extracted after simulations had reached a qualified coincidence (Current error function (EF): $\sim 5$ in ADS) between the experimental and simulated S-parameters. 
1992
Fig. 2 a The schematic
structure of microstrip
transmission line. b An
optical image of fabricated
microstrip transmission line
based on the epoxy coated-
CNF film

Fig. 3 An equivalent
circuit model of the
transmission line. The
parasitic elements $L, C$ and
$R$ were included in this

1992
Fig. 2 a The schematic
structure of microstrip
transmission line. b An
optical image of fabricated
microstrip transmission line
based on the epoxy coated-
CNF film

Fig. 3 An equivalent
circuit model of the
transmission line. The
parasitic elements $L, C$ and
$R$ were included in this

1992
Fig. 2 a The schematic
structure of microstrip
transmission line. b An
optical image of fabricated
microstrip transmission line
based on the epoxy coated-
CNF film

Fig. 3 An equivalent
circuit model of the
transmission line. The
parasitic elements $L, C$ and
$R$ were included in this

1992
Fig. 2 a The schematic
structure of microstrip
transmission line. b An
optical image of fabricated
microstrip transmission line
based on the epoxy coated-
CNF film

Fig. 3 An equivalent
circuit model of the
transmission line. The
parasitic elements $L, C$ and
$R$ were included in this

1992
Fig. 2 a The schematic
structure of microstrip
transmission line. b An
optical image of fabricated
microstrip transmission line
based on the epoxy coated-
CNF film

Fig. 3 An equivalent
circuit model of the
transmission line. The
parasitic elements $L, C$ and
$R$ were included in this

1992
Fig. 2 a The schematic
structure of microstrip
transmission line. b An
optical image of fabricated
microstrip transmission line
based on the epoxy coated-
CNF film

Fig. 3 An equivalent
circuit model of the
transmission line. The
parasitic elements $L, C$ and
$R$ were included in this

1992
Fig. 2 a The schematic
structure of microstrip
transmission line. b An
optical image of fabricated
microstrip transmission line
based on the epoxy coated-
CNF film

Fig. 3 An equivalent
circuit model of the
transmission line. The
parasitic elements $L, C$ and
$R$ were included in this
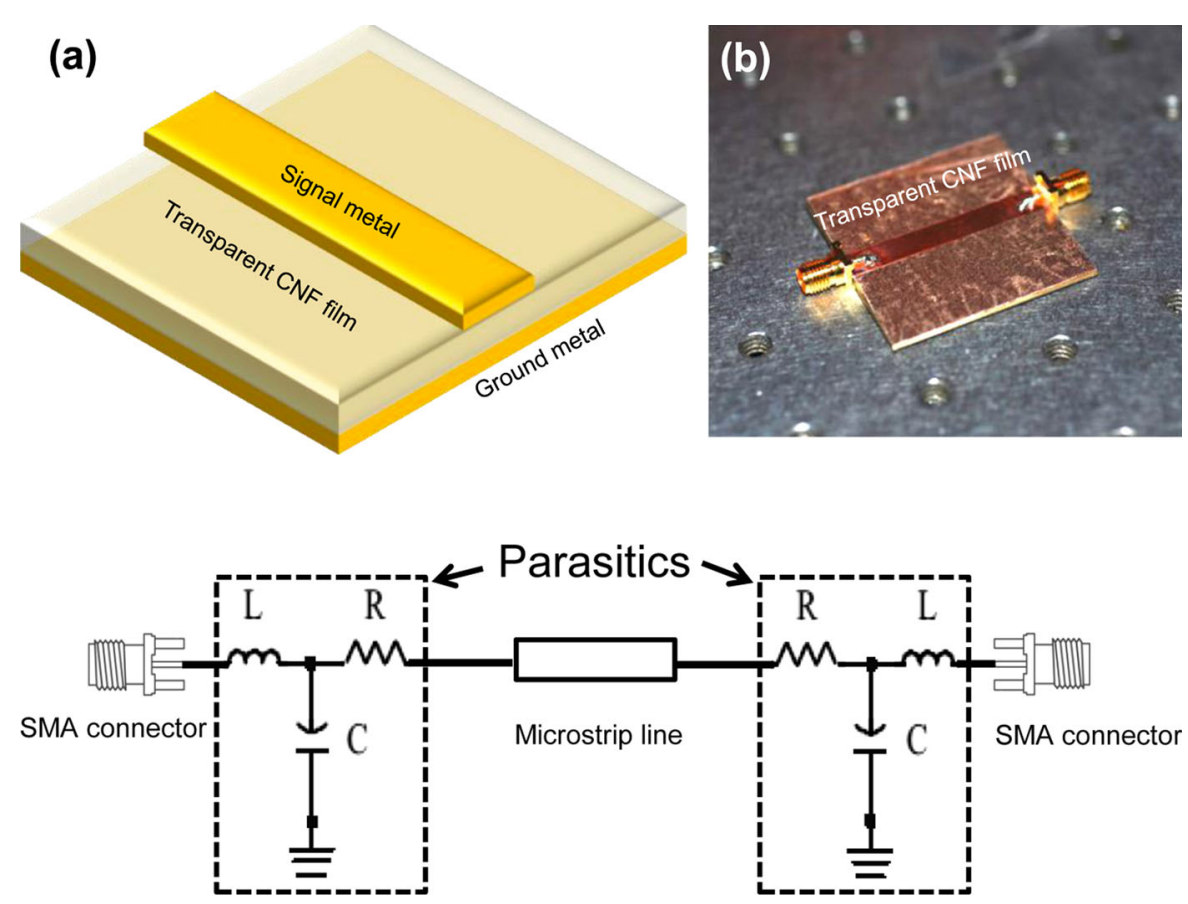
model
1992
Fig. 2 a The schematic
structure of microstrip
transmission line. b An
optical image of fabricated
microstrip transmission line
based on the epoxy coated-
CNF film

Fig. 3 An equivalent
circuit model of the
transmission line. The
parasitic elements $L, C$ and
$R$ were included in this

1992
Fig. 2 a The schematic
structure of microstrip
transmission line. b An
optical image of fabricated
microstrip transmission line
based on the epoxy coated-
CNF film

Fig. 3 An equivalent
circuit model of the
transmission line. The
parasitic elements $L, C$ and
$R$ were included in this

1992
Fig. 2 a The schematic
structure of microstrip
transmission line. b An
optical image of fabricated
microstrip transmission line
based on the epoxy coated-
CNF film

Fig. 3 An equivalent
circuit model of the
transmission line. The
parasitic elements $L, C$ and
$R$ were included in this

1992
Fig. 2 a The schematic
structure of microstrip
transmission line. b An
optical image of fabricated
microstrip transmission line
based on the epoxy coated-
CNF film

Fig. 3 An equivalent
circuit model of the
transmission line. The
parasitic elements $L, C$ and
$R$ were included in this

1992
Fig. 2 a The schematic
structure of microstrip
transmission line. b An
optical image of fabricated
microstrip transmission line
based on the epoxy coated-
CNF film

Fig. 3 An equivalent
circuit model of the
transmission line. The
parasitic elements $L, C$ and
$R$ were included in this

1992
Fig. 2 a The schematic
structure of microstrip
transmission line. b An
optical image of fabricated
microstrip transmission line
based on the epoxy coated-
CNF film

Fig. 3 An equivalent
circuit model of the
transmission line. The
parasitic elements $L, C$ and
$R$ were included in this model 


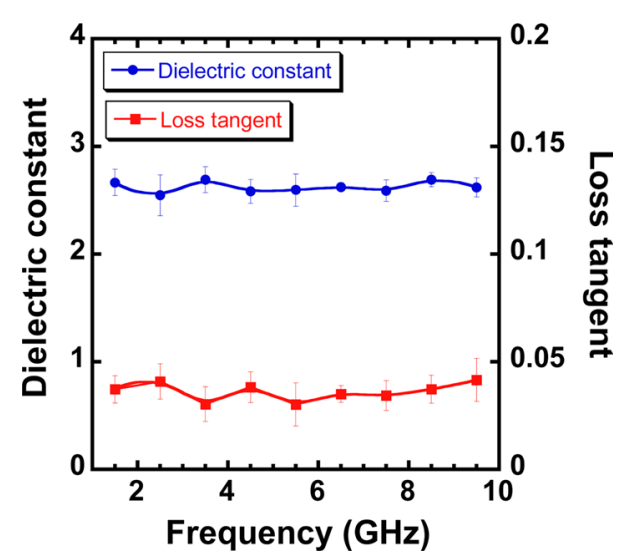

Fig. 5 Extracted microwave dielectric properties (dielectric constant and loss tangent) for the epoxy coated-CNF film

represents the mean value of results from the tested samples and the vertical error bars represent corresponding standard deviations. Mean value of the dielectric constant of the epoxy coated-CNF substrate is around 2.6 and mean value of loss tangent of the epoxy coated-CNF substrate is between 0.03 and 0.042 . These variations may come from differences in the fabrication processes of tested microstrip transmission lines. Because cellulose nanofibrils thin film can absorb moisture, the dielectric constant and loss tangent were investigated under various humidity conditions from 30 to $80 \%$. As shown in Fig. 6, the loss tangent value is significantly increased as the humidity level increased, particularly in the higher frequency regime, while the dielectric constant remained within a similar range. Such changes at the different humidities are attributed to the electro-

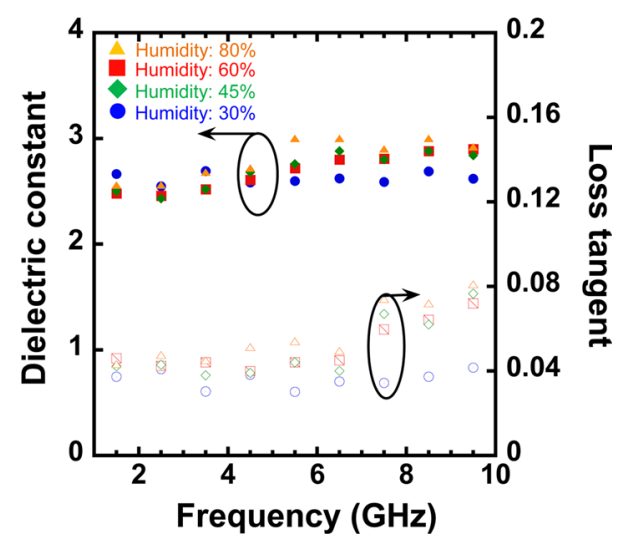

Fig. 6 Extracted microwave dielectric properties (dielectric constant and loss tangent) of the epoxy coated-CNF film at various humidity conditions from 30 to $80 \%$

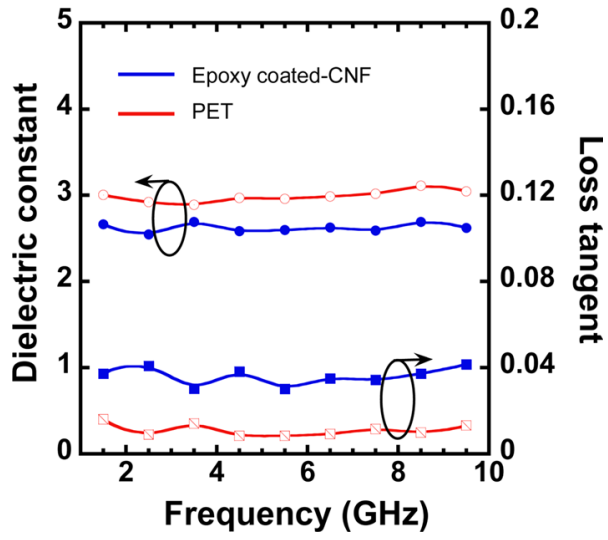

Fig. 7 Comparison of microwave dielectric properties for epoxy coated-CNF film and commercial PET film

magnetic polarization of the wood components in the CNF film, as well as the changes in the electrical conductance of the wood components which largely affect the loss tangent value. Therefore, microwave performance is dependent on the environmental humidity condition (James 1975; Torgovnikov 2012).

PET film has been previously employed in demonstrations of high speed flexible electronics (Sun et al. 2010a, b; Zhou et al. 2013; Cho et al. 2015) for its superior bendability and low cost. For comparison, microwave performance of a PET film was characterized in the frequency range of $1-10 \mathrm{GHz}$ using the same transmission line method. The PET film with a thickness of $254 \mu \mathrm{m}$ used in this study was supplied by the DuPont Company. Figure 7 shows the extracted average dielectric constant and loss tangent for both epoxy coated-CNF and PET films. The PET film shows an average dielectric constant around 3 and an average loss tangent between 0.008 and 0.01 in the frequency range of interest. The dielectric constant of CNF is slightly lower than that of PET film and the loss tangent of the epoxy coated-CNF film is slightly higher than that of PET film, but the differences are close. Hence, the epoxy coated-CNF film is suitable for high frequency applications, as we recently demonstrated (Jung et al. 2015; Seo et al. 2015). Considering the biodegradability of the epoxy coated-CNF film versus PET, employing the epoxy coated-CNF for flexible microwave applications is a viable approach to make environment-friendly electronics. The detailed measurement study shown in this work will also be useful for future microwave circuit designs. 


\section{Conclusions}

In summary, microwave dielectric and loss tangent properties of biodegradable epoxy coated-CNF thin film were characterized from 1 to $10 \mathrm{GHz}$. The dielectric constant of the epoxy coated-CNF is around 2.6 and loss tangent values range from 0.03 to 0.042 . Through comparison with PET film, the epoxy coated$\mathrm{CNF}$ is proven to be suitable for flexible microwave applications. The current study suggests that flexible epoxy coated-CNF film can be an environmentfriendly contender for flexible high speed RF device and circuitry applications composed of soluble metals, while serving as a self-disposable substrate to potentially reduce generation of electronic waste.

Acknowledgments The work was supported by AFOSR under a PECASE Grant FA9550-09-1-0482. The program manager is Dr. Gernot Pomrenke.

Open Access This article is distributed under the terms of the Creative Commons Attribution 4.0 International License (http:// creativecommons.org/licenses/by/4.0/), which permits unrestricted use, distribution, and reproduction in any medium, provided you give appropriate credit to the original author(s) and the source, provide a link to the Creative Commons license, and indicate if changes were made.

\section{References}

Abdul Khalil HPS, Bhat AH, Ireana Yusra AF (2012) Green composites from sustainable cellulose nanofibrils: a review. Carbohydr Polymers 87:963-979. doi:10.1016/j. carbpol.2011.08.078

Aguilar SM, Shea JD, Al-Joumayly MA et al (2012) Dielectric characterization of PCL-based thermoplastic materials for microwave diagnostic and therapeutic applications. IEEE Trans Biomed Eng 59:627-633. doi:10.1109/TBME.2011. 2157918

Baker-Jarvis J, Janezic M, DeGroot DC (2010) High-frequency dielectric measurements. IEEE Instrum Meas Mag 13:24-31. doi:10.1109/MIM.2010.5438334

Bledzki AK, Gassan J, Theis S (1998) Wood-filled thermoplastic composites. Mech Composite Mater 34:563-568. doi:10.1007/BF02254666

Chinga-Carrasco G (2011) Cellulose fibres, nanofibrils and microfibrils: the morphological sequence of MFC components from a plant physiology and fibre technology point of view. Nanoscale Res Lett 6:417. doi:10.1186/1556-276X-6-417

Cho S, Jung Y, Ma Z (2015) X-band compatible flexible microwave inductors and capacitors on plastic substrate. IEEE J Electron Devices Soc doi: 10.1109/JEDS.2015. 2446957
Couderc S, Ducloux O, Kim BJ, Someya T (2009) A mechanical switch device made of a polyimide-coated microfibrillated cellulose sheet. J Micromech Microeng 19:055006. doi:10. 1088/0960-1317/19/5/055006

Inui T, Koga H, Nogi M et al (2015) A miniaturized flexible antenna printed on a high dielectric constant nanopaper composite. Adv Mater 27:1112-1116. doi:10.1002/adma. 201404555

Isogai A, Saito T, Fukuzumi H (2011) TEMPO-oxidized cellulose nanofibers. Nanoscale 3:71-85. doi:10.1039/ C0NR00583E

James WL (1975) Dielectric properties of wood and hardboard: variation with temperature, frequency, moisture content, and grain orientation (No. FSRP-FPL-245). Forest products lab, Madison, Wisconsin

Jung YH, Chang T-H, Zhang H et al (2015) High-performance green flexible electronics based on biodegradable cellulose nanofibril paper. Nat Commun. doi:10.1038/ncomms8170

Lee H-Y (1995) Wideband characterization of a typical bonding wire for microwave and millimeter-wave integrated circuits. IEEE Trans Microw Theory Tech 43:63-68. doi:10. $1109 / 22.363006$

Lorenzen JA (2014) Green consumption and social change: debates over responsibility, private action, and access. Sociol Compass 8:1063-1081. doi:10.1111/soc4.12198

Minges ML (1989) Electronic materials handbook: packaging, vol. 1, ASM International, Materials Park, OH

Moon-Que Lee SN (1996) An accurate broadband measurement of substrate dielectric constant. Microw Guided Wave Lett IEEE 6:168-170. doi:10.1109/75.481077

Qing Y, Sabo R, Cai Z, Wu Y (2012) Resin impregnation of cellulose nanofibril films facilitated by water swelling. Cellulose 20:303-313. doi:10.1007/s10570-012-9815-0

Ramazani M, Miladi H, Shahabadi M, Mohajerzadeh S (2010) loss measurement of aluminum thin-film coplanar waveguide (CPW) lines at microwave frequencies. IEEE Trans Electron Devices 57:2037-2040. doi:10.1109/TED.2010. 2050110

Seo J-H, Ma Z, Zhou W (2014) Radio-frequency flexible electronics: transistors and passives. In: 2014 IEEE bipolar/ BiCMOS circuits and technology meeting (BCTM), pp 107-114

Seo J-H, Chang T-H, Lee J et al (2015) Microwave flexible transistors on cellulose nanofibrillated fiber substrates. Appl Phys Lett 106:262101. doi:10.1063/1.4921077

Sun L, Qin G, Huang H et al (2010a) Flexible high-frequency microwave inductors and capacitors integrated on a polyethylene terephthalate substrate. Appl Phys Lett 96:013509. doi:10.1063/1.3280040

Sun L, Qin G, Seo J-H et al (2010b) 12-GHz thin-film transistors on transferrable silicon nanomembranes for high-performance flexible electronics. Small 6:2553-2557. doi:10. 1002/smll.201000522

Thompson D, Tantot O, Jallageas H et al (2004) Characterization of liquid crystal polymer (LCP) material and transmission lines on LCP substrates from 30 to $110 \mathrm{GHz}$. IEEE Trans Microw Theory Tech 52:1343-1352. doi:10.1109/ TMTT.2004.825738

Torgovnikov G (2012) Dielectric properties of wood and woodbased materials. Springer Science \& Business Media, Berlin 
Yamashita E, Ohashi H, Atsuki K (1989) Characterization of microstrip lines near a substrate edge and design formulas of edge-compensated microstrip lines (MMICs). IEEE Trans Microw Theory Tech 37:890-896. doi:10.1109/22. 17456

Yun S-K, Lee H-Y (1995) Parasitic impedance analysis of double bonding wires for high-frequency integrated circuit packaging. IEEE Microw Guided Wave Lett 5:296-298. doi:10.1109/75.410403

Zhang K, Seo J-H, Zhou W, Ma Z (2012) Fast flexible electronics using transferrable silicon nanomembranes. J Phys Appl Phys 45:143001. doi:10.1088/0022-3727/45/14/ 143001
Zhang Y, Nypelö T, Salas C et al (2013) Cellulose nanofibrils. J Renew Mater 1:195-211. doi:10.7569/JRM.2013.634115

Zheng G, Cui Y, Karabulut E et al (2013) Nanostructured paper for flexible energy and electronic devices. MRS Bull 38:320-325. doi:10.1557/mrs.2013.59

Zhou H, Seo J-H, Paskiewicz DM et al (2013) Fast flexible electronics with strained silicon nanomembranes. Sci Rep. doi:10.1038/srep01291

Zou G, Gronqvist H, Starski JP, Liu J (2002) Characterization of liquid crystal polymer for high frequency system-in-apackage applications. IEEE Trans Adv Packaging 25:503-508. doi:10.1109/TADVP.2002.807593 